\title{
Joint salvage and biological repair of massive- cavity bone defects after extensive curettage of giant cell tumor around the knee with vascularized fibular autograft and cancellous allograft
}

\author{
Yun-Fa Yang ( $\nabla$ eyyangyunfa@scut.edu.cn ) \\ University of Technology

\section{Xiao-Sheng Gao} \\ Guangzhou First People's Hospital \\ Jian-Wei Wang \\ Guangzhou First People's Hospital \\ Zhong-He Xu \\ Guangzhou First People's Hospital
}

Guangzhou First People's Hospital, the Second Affiliated Hospital, School of Medicine, South China

\section{Research article}

Keywords: Giant cell tumor; Extensive Curettage; Joint salvage; Bone defect; Vascularized fibular graft

Posted Date: July 2nd, 2020

DOI: https://doi.org/10.21203/rs.3.rs-38293/v1

License: (c) (1) This work is licensed under a Creative Commons Attribution 4.0 International License.

Read Full License 


\section{Abstract}

Background: Treatment of giant cell tumor (GCT) around knee remains challenging because GCT is prone to recurrence and metastasis. Herein, we reported on our clinical experience with knee joint salvage and biological repair of massive-cavity bone defects after extensive curettage of GCT around the knee with vascularized fibular autograft and cancellous allograft in 12 patients.

Methods: All the patients underwent clinical evaluation, plain radiography and/or magnetic resonance imaging (MRI) of the knee right after admission. Their joint function was preserved, and the massivecavity bone defects were repaired by vascularized fibular autografts and cancellous allograft after extensive curettage of GCT around the knee. All the patients were evaluated through clinical examinations, plain radiography of the knee and chest, and Musculoskeletal Tumor Society (MSTS) scores of the lower extremity in the follow-ups.

Results: The follow-up duration ranged from 1.5 years to 12.0 years (mean 4.2 years). There were no local recurrences or lung metastasis in any of the 12 patients at the last follow-up. Ten patients had no pain or experienced occasional pain, and nine were able to resume their previous work. The mean range-ofmotion of knee flexion was $117^{\circ}$, and the extension was $-6^{\circ}$. The mean MSTS score was 24.7 , and a total of 10 patients had excellent or good MSTS scores.

Conclusion: Knee joint salvage and biological repair of massive-cavity bone defects could be achieved after extensive curettage with vascularized fibular autograft and cancellous allograft in patients with GCT around the knee.

\section{Background}

GCT is a relatively common type of primary benign tumor that usually occurs in the metaphyseoepiphyseal region of long bones [1]. It typically attacks young people (age $\leq 40$ ), and it shows no symptoms or only mild symptoms, which may last for weeks or even months before patients seek advice from an orthopedic surgeon. Some patients who suffer from GCT visit a doctor for the first time only because of the acute pain caused by pathological fractures [2]. Nevertheless, GCT can also be potentially aggressive and can metastasize. Consequently, in some GCT patients pulmonary metastases may be accidentally found only at their first clinical examination. Therefore, treatment of GCT located around the knee (the distal femur and proximal tibia) still remains quite challenging.

Currently, there are several methods that are used for the treatment of GCT around the knee, such as curettage or en-bloc resection with some reconstructive surgeries, arthrodesis by using a structural bone graft, a sandwich technique based on bone graft and polymethylmethacrylate (PMMA), and knee arthroplasty with structural bone allograft or knee prosthesis [3, 4]. However, each of these methods has its advantages and disadvantages, respectively. 
Intralesional curettage with PMMA or bone-graft fillings, which can preserve the knee joint function, is an effective primary treatment for GCT around the knee [5]. Yet, GCT has a relatively high recurrent rate after intralesional curettage $[3,4,6]$. Furthermore, GCT is usually associated with cortical invasion, extracompartmental extension, and pathologic fracture in the metaphyseoepiphyseal region of the knee. Therefore, in order to effectively treat GCT around the knee, unitary intralesional curettage may not be the optimal choice.

Knee arthrodesis reconstructed by structural bone allograft or autograft after En-bloc resection of GCT is not recommended since most of these GCT patients are young and active, and wish to retain a functional knee [7-10]. Moreover, structural bone grafts may also lead to certain complications, including fracture, nonunion, infection, GCT recurrence, and donor complications associated with non-vascularized fibular autograft [11].

Therefore, the knee arthroplasty with allograft or prosthesis after En-bloc resection of GCT may lead to much better clinical outcomes compared to arthrodesis. Still, due to nonunion between allograft and host caused by insufficient blood supply, infection, bone allograft absorption or fracture, and loosening of prosthesis promoted by non-biological repair, the knee arthroplasty with structural bone allografts or knee prosthesis may not always be suitable for the treatment of GCT located around the knee, especially in the young and active patients.

It is possible to preserve knee joint function by using a sandwich technique that adopts bone grafts and PMMA to fill the bone cavity after extensive curettage, which may incorporate, remodel, and preserve subchondral bone stock, thus maintaining the knee joint function [12-14]. However, the sandwich technique may accelerate degenerative knee osteoarthritis followed with articular cartilage damage resulting in thermal effect or other side effects of PMMA cement [12].

To overcome these shortcomings, we proposed a method that combines the advantages of the sandwich technique, extensive curettage technique, and microsurgical technique for the treatment of GCT around the knee. We effectively achieved local tumor control, knee joint salvage, and biological repair of massivecavity bone defects after extensive curettage with vascularized fibular autograft and cancellous allografts in patients with GCT around the knee. Herein, we presented the clinical outcomes of this treatment.

\section{Methods}

This study was approved by the ethics committee of Guangzhou First People's Hospital. After hospitalization, patients suffering from histologically confirmed GCT (Campanacci grade II or III) located around knee were well informed of this study. A total of 12 patients who were treated at our department between 2004 and 2016 were enrolled in the study. In order to preserve knee joint and biologically repair massive-cavity bone defects after extensive curettage of GCT, 12 patients were treated by using vascularized fibular autograft and cancellous allograft. 


\section{Patients}

Among 12 enrolled patients, 9 were men and 3 women, with an age range from 18 to 46 years (mean age was 28.2 years). All 12 patients underwent knee plain radiography and/or magnetic resonance imaging (MRI) as the initial assessment after hospitalization. Chest X-rays and/or chest computed tomography (CT) scans were initially used during the first evaluation. There were no patients with lung metastases at the time of the enrollment time in this study. Five patients had GCT lesion located in the left knee and seven patients in the right knee. Follow-ups in the duration of at least 18 months were arranged for all 12 patients.

\section{Surgical Technique}

\section{Extensive Curettage of the GCT}

In order to avoid contamination of tumor cells, we strictly abided by the principle of tumor resection and also took good care of the surrounding tissues during extensive curettage of GCT.

Firstly, a bone window was carefully opened in the GCT site, after which GCT was removed through the window. In order to completely resect the GCT, a high-speed burr was used in different directions in order to remove $2 \mathrm{~mm}$ bone from the inner surface of the bone cavity until the normal bone was visible. Finally, the bone cavity was cleaned, and the extensive curettage was completed. Usually, $2 \%$ iodine tincture was smeared in the bone cavity for about 2 minutes, after which $75 \%$ alcohol was used to remove iodine, and lastly irrigation with $0.9 \%$ saline was performed for cavity cleaning. During this step, we carefully kept the surrounding tissues from coming in contact with iodine tincture. In fact, iodine tincture was not used in the tumor cavity if pathological fracture existed.

After the extensive curettage, the size of bone cavity defects was attentively measured in order to prepare the vascularized fibular autograft.

\section{Harvesting of vascularized fibular graft}

The fibula was harvested according to references 15 and $16[15,16]$. In order to effectively avoid tumor cell contamination, the contralateral fibula was selected as donor.The length of the fibular graft was affirmed according to the bone defect secondary to the extensive curettage of GCT. Actually, the length of the fibular graft should almost be two times of the defects because fibular graft may need to be folded to repair the bone cavity defects.

\section{Repair of massive-cavity bone defects after extensive curettage}

The fibula (or folded fibula) and cancellous allograft were transplanted to fill the massive-cavity bone defects after GCT extensive curettage. Firstly, the fibular graft was inserted in the cavity, and the 
appropriate length of the graft was confirmed by using intraoperative C-arm X-ray examination. Next, the cancellous allograft was used to fill the remaining bone cavity after fibular insertion. Plate, screw, or kwire were chosen for skeletal fixation. The peroneal artery and veins were anastomosed via an end-to-end and/or end-to-side anastomosis with the popliteal artery or anterior tibial artery and veins, or one of the accompanying veins and the great saphenous vein. We closed the incision after we affirmed the blood supply of soft tissue attached around the fibula is sufficient after bone grafts transplantation.

\section{Postoperative Assessment}

The knee was usually fixed in a functional position ( $15^{\circ}-20^{\circ}$ of knee flexion) with a plaster splint after surgery. Knee range-of-motion exercises were started at 3-6 weeks, and partial weight-bearing started at 6 weeks postoperatively. The splint was removed within 3 to 6 weeks, based on knee stability. After splint was removed, the patients were allowed to continue partial weight-bearing with crutches.

Patients were allowed to bear full weight according to their tolerance 3 to 4 months after repair operation when radiographs showed the evidence of healing between the vascularized fibular graft ends and host bone.

Patients were followed-up at 1,2, and 3 months postoperatively, then every 3 months till 18 months after surgery, and every year if possible. Plain AP and lateral radiographs were taken at every follow-up.

The bone healing was tracked by comparing radiographs taken in follow-ups. The bone was considered healed when the bridging callus appeared across fibular ends and host bone in radiographs.

The knee function was routinely evaluated at the follow-up clinical examination and after 18 months postoperatively by using the revised 30-point functional evaluation system for lower extremity advocated by the Musculoskeletal Tumor Society (MSTS), which addressed pain, functional situation, emotional acceptance, usage of external support, walking ability and gait condition. Each variable was calculated on a 5-point scale [17].

Complications such as local recurrences, infection, fractures, subchondral collapse, knee joint narrowing, and lung metastases were assessed. The complications in the donor leg were also recorded. Sometimes, all the internal fixations were removed 18 months after surgery, if necessary.

\section{Results}

The duration of follow-up ranged from 1.5 years to 12.0 years, with an average of 4.2 years. Bone union was found in all the 12 patients within 3 and 5 months after repair surgery. No patients experienced GCT recurrence by the time of the last follow-up. There was no evidence of lung metastases in any of the patients. One patient (Campanacci III GCT) had knee joint narrowing and fibula donor site discomfort, while no patient complained of joint instability of the knee or ankle. There were no postoperative infections, fractures, subchondral collapses, or neurovascular complications in any of the 12 patients. 
Six patients (50.0\%) were pain-free, 4 (33.3\%) experienced mild and occasional pain, 2 (16.7\%) had moderate but tolerable pain, and no patient complained of intolerable pain. Four (33.3\%) patients continued employment at their previous place of work without any restriction, five (41.7\%) came back to work with little limitations, $3(25.0 \%)$ had the ability to work, but they did not want to continue work, and no patient was disabled due to the GCT after the reconstructive microsurgery.

All the 12 patients showed some limitations in knee rang-of-motion. Nine patients $(75.0 \%)$ showed the knee flexion ranging from $90^{\circ}$ to $120^{\circ}, 3$ patients $(25.0 \%)$ had the knee flexion ranging from $120^{\circ}$ to $135^{\circ}$. No patient had a flexion restricted in less than $90^{\circ}$. The mean range of flexion was $117^{\circ}$, and the extension was $-6^{\circ}$ in the 12 patients.

According to the MSTS scores of the lower extremity, 3 patients (25.0\%) had excellent outcomes, 7 (58.3\%) good, 1 (8.3\%) moderate, and 1 (8.4\%) poor. The mean MSTS score was 24.7 , and totally, 10 patients $(83.3 \%)$ had excellent or good MSTS scores.

[Figures (Fig. 1; Fig. 2)]

\section{Discussion}

The principal of GCTs treatment should be based on completely removing the tumor, reducing recurrence, and preserving or recovering limb function $[3,4,18]$. In this study, clinical examination revealed no cases of lung metastasis or local GCT recurrence at the last follow-up. The mean MSTS score was 24.7; 10 patients (83.3\%) had excellent or good MSTS scores. Our results revealed that it was possible to preserve knee joint and biologically repair massive-cavity bone defects after extensive curettage with vascularized fibular autograft and cancellous allograft in patients with GCT around the knee.

\section{Extensive curettage can reduce the risk of recurrence}

GCT is an aggressive benign tumor with a high local recurrent rate that can occur after the curettage or cementing of GCT located around the knee [19-21]. Moreover, recurrence is highly correlated with metastasis, which can be reduced by early and complete tumor resection. Therefore, the reduction of GCT recurrence is of great importance.

Tumor cells remaining or being implanted in the surgical site during surgery usually causes local recurrence of GCT [22]. Previous studies have shown that en-bloc resection of GCT is very effective in reducing recurrence [23]. However, when the GCT is located around the knee, en-bloc resection is not recommended if we want to save the knee joint. Otherwise, the knee must be reconstructed by the prosthesis. Therefore, extensive curettage may be more suitable for reducing local tumor recurrence and knee salvage, especially when using high-speed burr for complete removal of GCT during curettage surgery [24]. Actually, extensive curettage has already been shown to have successful outcomes in the prevention of GCT recurrence [25]. 


\section{Vascularized fibula can repair massive-cavity bone defects and save knee joint}

Many reconstruction procedures, including sandwich technique, arthroplasty, osteoarticular allograft, allograft arthrodesis, and vascularized or non-vascularized fibular autograft with or without arthrodesis have been proposed after wide resection of GCT around the knee [3, 4, 25-27].

Sandwich technique with extensive curettage has shown to prevent tumor recurrence and lead to satisfactory knee salvage [25]. However, since the sandwich technique requires PMMA, which cannot repair bone defects biologically and may lead to thermal injuries, it may not be the optimal method for all GCT around the knee, especially in young and active patients [5, 28-31].

Because of the incompatibility between the prostheses and the host bone, prostheses may not be suitable for long-term survival, which might be ensured by bony union. Currently, there is still a lack of evidence on the complete biological union between the host bone and prostheses. At the same time, it has been shown that bone graft can usually achieve complete bone union. Thus, bone grafts are still the reliable choice for repair of bone cavity defects secondary to GCT extensive curettage in young patients.

All bone allografts have disadvantages, including potential immunologic reactions, lack of blood supply and osteogenic cells, and the possibility of joint collapse secondary to bone allograft absorption. As a result, simply, bone allografts are not suitable for knee joint salvage.

Non-vascular autografts have many advantages since they contain normal bone structure and are free from disease transmissions. However, these grafts may also have some complications (such as nonunion, donor complications), which may eventually lead to unsatisfactory outcomes [23]. Since some cases of our patients presented with the non-vascular fibular autografts collapse in wrist and knee reconstruction, we did not repair massive bone defects only by using non-vascular fibular grafts.

Vascularized fibular autografts have the same donor complications as non-vascularized ones. Yet, vascularized fibular autografts contain living bone cells and an intrinsic blood supply system, which maintain the ability of osteogenesis after transplantation. Their biological advantage is that the healing process between the host and the fibular bone autograft is identical to that of normal fracture healing because there are no immunologic reactions and creeping substitution. Therefore, the union time for these grafts is much shorter (as short as 3-5 months) than that of non-vascularized bone grafts or allografts. Hence, the vascularized fibula combined with cancellous allografts may be a suitable method for young patients who desire long-term knee joint salvage after GCT extensive curettage.

\section{Strengths and weaknesses}

Our method combined the advantages of the sandwich technique, extensive curettage technique, and microsurgical technique. In order to achieve joint salvage and biological repair, bone grafts, especially 
vascularized fibular autograft, were used in the place of PMMA of sandwich technique to fill the bone cavity defects after extensive curettage.

Although our results indicated that joint salvage and biological repair of massive-cavity bone defects after extensive tumor curettage with vascularized fibular autograft and cancellous allograft for GCT around the knee are feasible, effective, and reliable, there are still some limitations in our study. Firstly, the operation procedure is so complicated that it could not be completely performed without microsurgical skills and techniques. Secondly, since the sample size was small, the study findings need to be further confirmed by additional clinical evidence, including longer clinical assessment. Thirdly, knee function may be severely limited after the joint salvage because the knee should be immobilized for at least 3 weeks.

\section{Conclusion}

It is reliable to achieve knee joint salvage and biological repair of massive-cavity bone defects after extensive curettage with vascularized fibular autograft and cancellous allograft in patients with GCT around the knee.

\section{List Of Abbreviations}

giant cell tumor (GCT); polymethylmethacrylate (PMMA); magnetic resonance imaging (MRI); computed tomography (CT); musculoskeletal tumor society (MSTS).

\section{Declarations}

\section{Ethics approval and consent to participate}

The study was approved by the Ethics Committee of Guangzhou First People's Hospital.

\section{Consent for publication}

Not applicable.

\section{Availability of data and materials}

The dataset supporting the conclusions of this manuscript is available upon request by contacting the corresponding author.

\section{Competing interests}

None of the authors has competing interests in any of the products, devices, or drugs mentioned in this manuscript.

\section{Funding}


This study was partially supported by Guangzhou planned project of science and technology (No. 201707010261).

\section{Authors' contributions}

YFY and ZHX performed study and assembled data. XSG and JWW participated in this study and assisted in drafting the manuscript. YFY designed the study, performed data analysis, and drafted the manuscript.

\section{Acknowledgements}

We gratefully acknowledge Guangzhou planned project of science and technology (No. 201707010261) for its partial support, and L Li for his C-arm X-ray assistance during reconstructive surgery.

\section{References}

1. Turcotte RE. Giant cell tumor of bone. Orthop Clin North Am. 2006, 37(1): 35-51.

2. Murphey MD, Nomikos GC, Flemming DJ, Gannon FH, Temple HT, Kransdorf MJ. From the archives of AFIP. Imaging of giant cell tumor and giant cell reparative granuloma of bone: Radiologicpathologic correlation. Radiographics. 2001, 21(5):1283-309.

3. Rigollino AV, Fernando TS, Tanaka MH, Souza MM. Giant cell tumor locally advanced around the knee: treatment and literature review. Rev Bras Ortop. 2017;52(4):473-8.

4. Montgomery C, Couch C, Emory CL, Nicholas R. Giant Cell Tumor of Bone: Review of Current Literature, Evaluation, and Treatment Options. J Knee Surg. 2019;32(4):331-6.

5. van der Heijden L, van de Sande MA, Heineken AC, Fiocco M, Nelissen RG, Dijkstra PD. Mid-term outcome after curettage with polymethylmethacrylate for giant cell tumor around the knee: higher risk of radiographic osteoarthritis? J Bone Joint Surg Am. 2013;95(21):e159.

6. Arbeitsgemeinschaft Knochentumoren, Becker WT, Dohle J, Bernd L, Braun A, Cserhati M, Enderle A, Hovy L, Matejovsky Z, Szendroi M, Trieb K, Tunn PU. Local recurrence of giant cell tumor of bone after intralesional treatment with and without adjuvant therapy. J Bone Joint Surg Am. 2008;90(5):1060-7.

7. Lv C, Tu C, Min L, Duan H. Allograft arthrodesis of the knee for giant cell tumors. Orthopedics. 2012;35(3):e397-402.

8. Saikia KC, Bhuyan SK, Saikia SP, Rongphar R, Jitesh P. Resection and arthrodesis of the knee joint for giant cell tumours of bone. J Orthop Surg (Hong Kong). 2010;18(2):208-14.

9. Kapoor SK, Tiwari A. Resection arthrodesis for giant cell tumors around the knee. Indian J Orthop. 2007;41(2):124-8.

10. Bassiony AA, Abdelrahman M, Abdelhady A, Assal MK. Resection arthrodesis for the management of aggressive giant cell tumor of the distal femur. Indian J Orthop. 2009;43(1):67-71. 
11. Donati D, Giacomini S, Gozzi E, Salphale Y, Mercuri M, Mankin HJ, Springfield DS, Gebhardt MC. Allograft arthrodesis treatment of bone tumors: a two-center study. Clin Orthop Relat Res. 2002; (400):217-24.

12. Kundu ZS, Gogna P, Singla R, Sangwan SS, Kamboj P, Goyal S. Joint salvage using sandwich technique for giant cell tumors around knee. J Knee Surg. 2015;28(2):157-64.

13. Saibaba B, Chouhan DK, Kumar V, Dhillon MS, Rajoli SR. Curettage and reconstruction by the sandwich technique for giant cell tumours around the knee. J Orthop Surg (Hong Kong). 2014;22(3):351-5.

14. Salgia A, Biswas S, Agrawal R, Goyal V. Multicentric giant cell tumor around the knee. Indian J Orthop. 2007;41(2):151-3.

15. Yang YF, Zhang GM, Xu ZH, Wang JW. Homeochronous usage of structural bone allograftswith vascularized fibular autografts for biological repair of massive bone defects in the lower extremities after bone tumor excision. J Reconstr Microsurg. 2010, 26(2):109-15.

16. Yang YF, Zhang GM, Huo ZQ, Xu ZH, Xu DC. Reconstruction of the distal ulnar epiphysis with vascularized proximal fibula including epiphysis in children after osteochondroma resection: report of two cases. Plast Reconstr Surg. 2013, 132(5):784e-9e.

17. Enneking WF, DunhamW, Gebhardt MC,MalawarM, Pritchard DJ. A system for the functional evaluation of reconstructive procedures after surgical treatment of tumors of the musculoskeletal system. Clin Orthop Relat Res. 1993;(286):241-6

18. Ayerza MA, Aponte-Tinao LA, Farfalli GL, Restrepo CA, Muscolo DL. Joint preservation after extensive curettage of knee giant cell tumors. Clin Orthop Relat Res. 2009;467(11):2845-51.

19. Lausten GS, Jensen PK, Schiødt T, Lund B. Local recurrences in giant cell tumour of bone. Long-term follow up of 31 cases. Int Orthop. 1996;20(3):172-6.

20. Remedios D, Saifuddin A, Pringle J. Radiological and clinical recurrence of giant-cell tumour of bone after the use of cement. J Bone Joint Surg Br. 1997;79(1):26-30.

21. Pettersson $H$, Rydholm A, Persson B. Early radiologic detection of local recurrence after curettage and acrylic cementation of giant cell tumours. Eur J Radiol. 1986;6(1):1-4.

22. Saiz P, Virkus W, Piasecki P, Templeton A, Shott S, Gitelis S. Results of giant cell tumor of bone treated with intralesional excision. Clin Orthop Relat Res. 2004, (424):221-6.

23. Yang YF, Wang JW, Huang $P, X u Z$ ZH. Distal radius reconstruction with vascularized proximal fibular autograft after en-bloc resection of recurrent giant cell tumor. BMC Musculoskelet Disord. 2016;17(1):346.

24. Algawahmed H, Turcotte R, Farrokhyar F, Ghert M. High-speed burring with and without the use of surgical adjuvants in the intralesional management of giant cell tumor of bone: a systematic review and meta-analysis. Sarcoma. 2010;2010:pii: 586090.

25. Banerjee S, Sabui KK, Chatterjee R, Das AK, Mondal J, Pal DK. Sandwich reconstruction technique for subchondral giant cell tumors around the knee. Curr Orthop Pract. 2012;23(5):459-66. 
26. Muscolo DL, Ayerza MA, Aponte-Tinao LA, Ranalletta M. Use of distal femoral osteoarticular allografts in limb salvage surgery. J Bone Joint Surg Am. 2005;87(11):2449-55.

27. Mankin HJ, Hornicek FJ. Treatment of giant cell tumors with allograft transplants: a 30-year study. Clin Orthop Relat Res. 2005;439(439):144-50.

28. Leeson MC, Lippitt SB. Thermal aspects of the use of polymethylmethacrylate in large metaphyseal defects in bone. A clinical review and laboratory study. Clin Orthop Relat Res. 1993;(295): 239-45.

29. Mjöberg B, Pettersson $H$, Rosenqvist R, Rydholm A. Bone cement, thermal injury and the radiolucent zone. Acta Orthop Scand. 1984; 55(6):597-600.

30. Nelson DA, Barker ME, Hamlin BH. Thermal effects of acrylic cementation at bone tumour sites. Int J Hyperthermia. 1997; 13(3):287-306.

31. von Steyern FV, Kristiansson I, Jonsson K, Mannfolk P, Heinegård D, Rydholm A. Giant-cell tumour of the knee: the condition of the cartilage after treatment by curettage and cementing. J Bone Joint Surg Br. 2007;89(3):361-5.

\section{Figures}

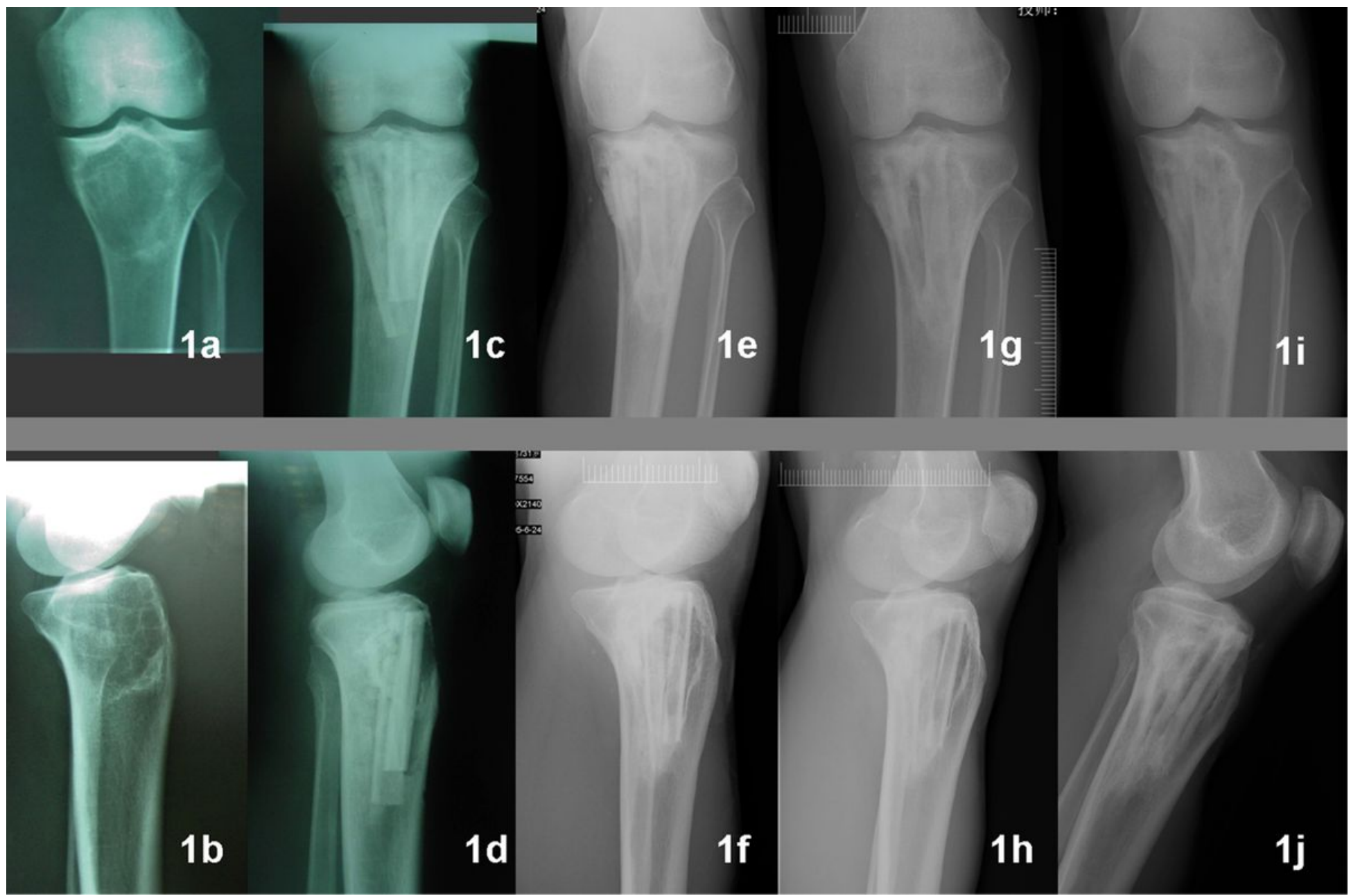

Figure 1 
Anteroposterior and lateral X-ray of a 29-year-old man with GCT of the left knee (Campanacci grade II). $(a, b)$ Before operation; (c-j) 1 week (1c, 1d), 1 year $(1 \mathrm{e}, 1 \mathrm{f}), 2$ years $(1 \mathrm{~g}, 1 \mathrm{~h})$, and 6 years $(1 \mathrm{i}, 1 \mathrm{j})$ after microsurgical repair.

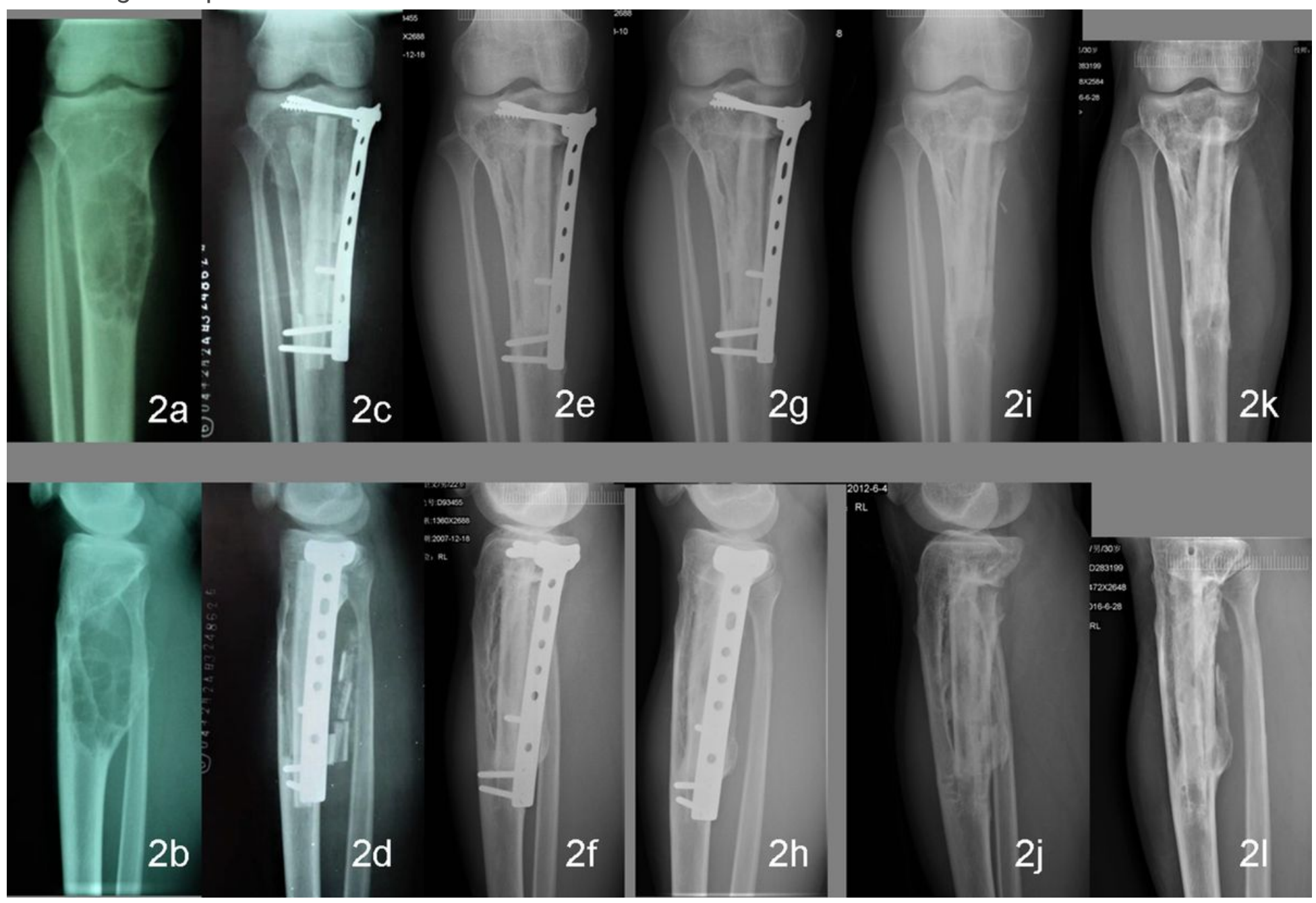

\section{Figure 2}

Anteroposterior and lateral X-ray of a 18-year-old man with GCT of the right knee (Campanacci grade III). $(a, b)$ Before operation; (c-l) 1 week (2c, 2d), 3 years $(2 e, 2 f), 5$ years $(2 g, 2 h), 8$ years $(2 i, 2 j)$, and 12 years $(2 \mathrm{k}, 2 \mathrm{l})$ after microsurgical reconstruction. 